\title{
Years off Your Life? The Effects of Homicide on Life Expectancy by Neighborhood and Race/Ethnicity in Los Angeles County
}

\author{
Matthew Redelings, Loren Lieb, and Frank Sorvillo
}

\begin{abstract}
Homicide is one of the leading causes of death in Los Angeles County and is known to be elevated in low-income urban neighborhoods and in black males. However, because homicide occurs primarily among young adults, mortality rate statistics may underrepresent its importance. We estimated the impact of homicide on life expectancy by demographic group and geographic area in Los Angeles County, 2001-2006. Life expectancy estimates were calculated using mortality records and population estimates for Los Angeles County. Cause elimination techniques were used to estimate the impact of homicide on life expectancy. Homicide was estimated to reduce life expectancy by 0.4 years for Los Angeles County residents and by 2.1 years for black males. The impact of homicide on life expectancy was higher in low-income neighborhoods. In some low-income urban neighborhoods, homicide was estimated to decrease life expectancy in black males by nearly 5 years. Homicide causes substantial reductions in life expectancy in Los Angeles County. Its impact is magnified among black males and in low-income urban areas, underscoring the need for homicide reduction in urban centers.
\end{abstract}

KEYWORDS Homicide, Life expectancy, Urban health

Like many counties with large urban areas, Los Angeles County (LAC) has a high homicide rate, particularly in low-income urban neighborhoods and among black males. ${ }^{1}$ Homicide is the tenth most common cause of death in LAC; however, this fact alone obscures its true impact. ${ }^{1}$ Homicide tends to affect young adults and often results in premature death, which is not adequately represented by providing raw numbers or mortality rates. Homicide is the second leading cause of premature death in LAC as measured in years of potential life lost (YPLLs) before age $75 .{ }^{1}$ Evaluation of the impact of homicide on life expectancy offers a unique way of assessing its mortality burden which (unlike YPLL calculations) is able to account for population trends and for the risk of death from competing causes. ${ }^{2} \mathrm{We}$ examined homicide's impact on life expectancy by geographic area and demographic group in Los Angeles County during 2001-2006.

\section{METHODS}

Life expectancy was calculated using methods described by the National Center for Health Statistics. ${ }^{3}$ Birth and mortality files were used in conjunction with Population

Redelings, Lieb, and Sorvillo are with the Los Angeles County Department of Public Health, Data Collection and Analysis Unit, Los Angeles, CA, USA.

Correspondence: Matthew Redelings, MPH, Los Angeles County Department of Public Health, Data Collection and Analysis Unit, Los Angeles, CA, USA. (E-mail: mredelings@ph.lacounty.gov) 
Estimates and Projection System (PEPS) population estimates for life expectancy calculations. ${ }^{4}$ Projected life expectancy if homicide were reduced was calculated after adjusting population counts and numbers of deaths to reflect the number of homicides that could have been prevented. ${ }^{2,5,6}$ The number of homicides that could have been prevented was estimated by multiplying the total number of homicides by a reduction factor (in our tables: $30 \%, 50 \%$, and 100\%), then adjusted for the possibility that individuals whose deaths from homicide were prevented would have died instead from other causes. The probability of death from other causes was calculated by examining mortality rates from other causes of death in individuals of the same demographic/geographic group. Age-adjusted mortality rates were standardized to the 2000 US population. ${ }^{7}$ Homicide deaths were defined as deaths for which the ICD codes U01-U02, X85-Y09, or Y87.1 were recorded as the underlying cause of death.

LAC is divided into eight service planning areas (SPAs) which are subdivided into 26 health districts. Geographical comparisons were conducted at the health district and SPA level. Federal poverty level data by health district and SPA were obtained from PEPS. ${ }^{3}$ Linear regression was used to examine the relationship between neighborhood poverty levels and the impact of homicide on life expectancy. Because homicide rates tend to be elevated in low-income urban neighborhoods, ${ }^{1}$ special attention was paid to the four health districts which make up South SPA, in urban Los Angeles. A map of health districts and SPAs is available at: http://lapublichealth.org/epi/docs/spahd_2002.pdf.

\section{RESULTS}

Life expectancy in LAC during 2001-2006 was 79.5 years. Life expectancy was reduced in males and in blacks (Table 1) and varied by health district (Figure 1). Regression analyses suggested a modest linear relationship between life expectancy and percent of the population below the federal poverty level $\left(\beta=-0.21, r^{2}=0.46\right.$, $p<0.001)$. Poverty levels were elevated in South SPA compared to LAC as a whole $(29.2 \%$ versus $16.4 \%)$. South SPA also had a higher percentage of black $(33.8 \%)$ and Latino $(62.0 \%)$ residents than LAC (respectively, 9.4\% and $46.2 \%$ ).

The age-adjusted homicide rate during the study period was 10.6 deaths per 100,000 population, with 6,491 deaths reported. The majority of homicide deaths $(82.4 \%)$ occurred among persons $15-44$ years of age. Homicide rates were elevated in blacks and in males and were higher in South SPA than in other SPAs. Eighty-four percent of all homicide deaths in males and in $52 \%$ of all homicide deaths in females were firearm-related. In South SPA, 92\% of homicide deaths in black males were firearm-related.

The impact of homicide on life expectancy was elevated in blacks and in males and differed by health district. Linear regression showed an exponential relationship (Figure 1) between the percentage of the population below the poverty level in each health district and the years of life expectancy lost due to homicide $(\beta=0.07$, $\left.r^{2}=0.74, p<0.001\right)$ where the impact of homicide on life expectancy nearly doubled (a 97\% increase was predicted) for each 10\% increase in percentage of the population below the poverty level. Homicide was estimated to reduce the life expectancy of black males by 2.1 years in LAC and by nearly 5 years in lowincome urban areas such as South Health District (Table 2).Even a 30\% reduction in homicide might have increased the life expectancy of black males in urban health districts by over 1 year. 


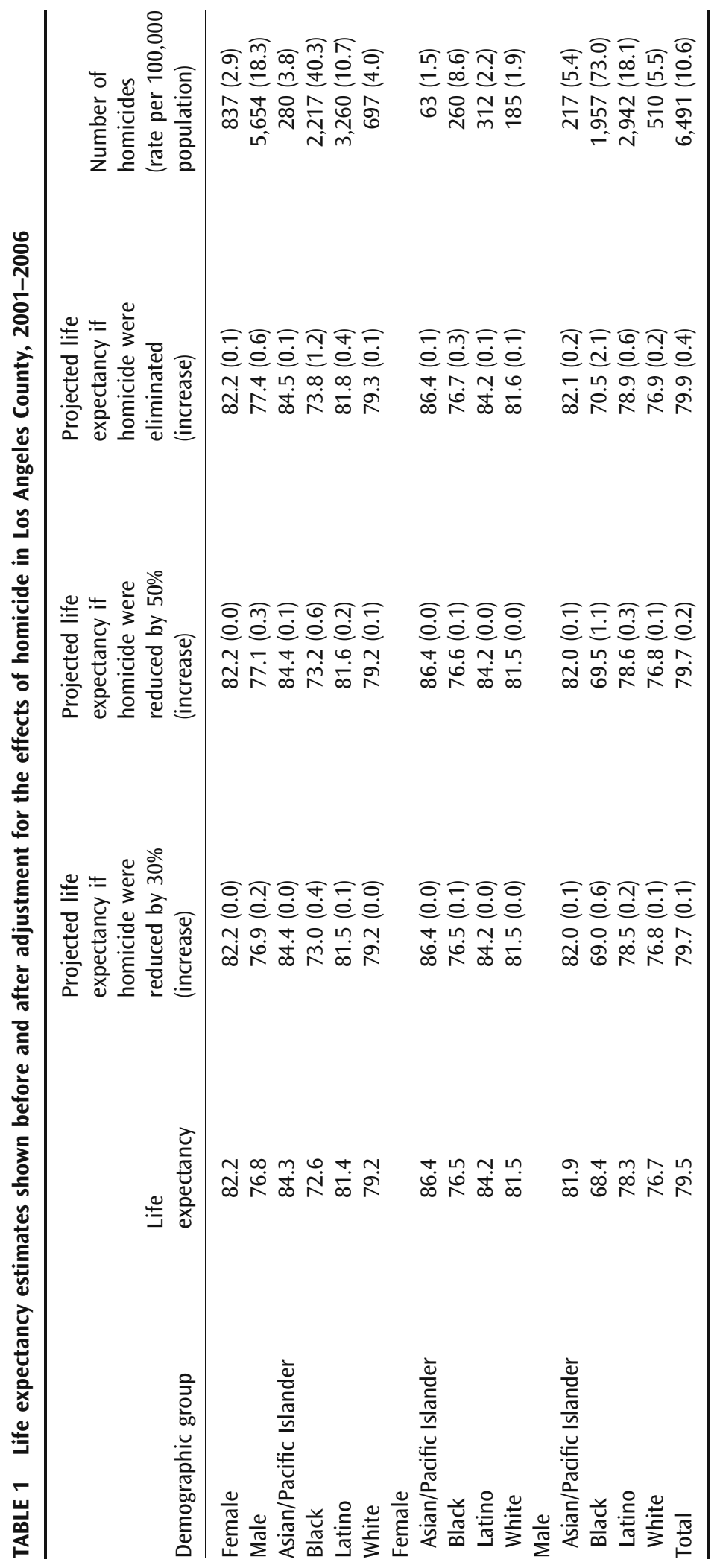




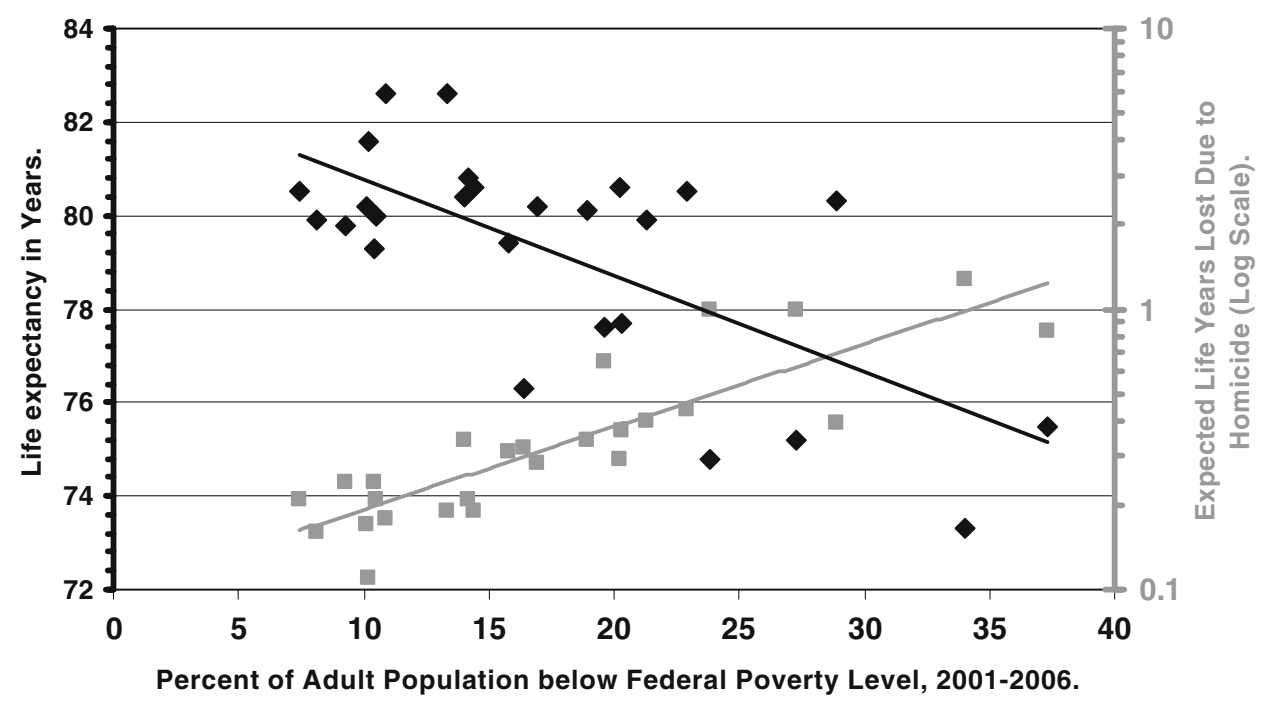

FIGURE 1. Poverty levels by Health District in Los Angeles County compared with life expectancy in years and expected life years lost due to homicide, 2001-2006.

\section{DISCUSSION}

Homicide causes substantial reductions in life expectancy in Los Angeles County. The impact of homicide on life expectancy is magnified among black males and in low-income urban areas, exacerbating racial and geographic disparities in life expectancy which would exist even if homicide were not a factor. This underscores the importance of effective homicide reduction strategies in LAC. Even modest decreases in homicide rates could cause substantial gains in life expectancy for black males in urban Los Angeles.

Homicide reduction efforts in LAC should place special emphasis on preventing homicides in young black males in low-income urban neighborhoods. Because the large majority of these deaths are firearm-related, prevention efforts should include enforcement of existing gun laws and entail efforts to reduce gun availability and ownership. Numerous other strategies have also been suggested, including increased policing and prosecution, counseling for at-risk youth, gang truces and anti-gang initiatives, and after-school programs for adolescents. ${ }^{8-10}$

Substantial success in reducing homicide rates is possible. Boston's Operation Ceasefire coincided with a $63 \%$ reduction in youth homicides in Boston. ${ }^{11}$ Operation Ceasefire focused on reducing trafficking of illicit firearms and on a mixture of focused policing and social services for gang members to discourage violence and encourage healthy alternatives. ${ }^{11}$ While LAC differs from Boston, recent trial efforts have met some success in replicating Operation Ceasefire's success in selected LAC neighborhoods. ${ }^{12}$

High homicide rates are often correlated with low levels of social cohesion in the community, and multiple factors in the social environment have been described as antecedents to homicide and violent crime. ${ }^{13-18}$ In our data, $75 \%$ of the variation by neighborhood in homicide's impact on life expectancy could be predicted by examining neighborhood poverty levels. This underscores the importance of addressing economic inequities as a part of homicide prevention efforts. Communities 


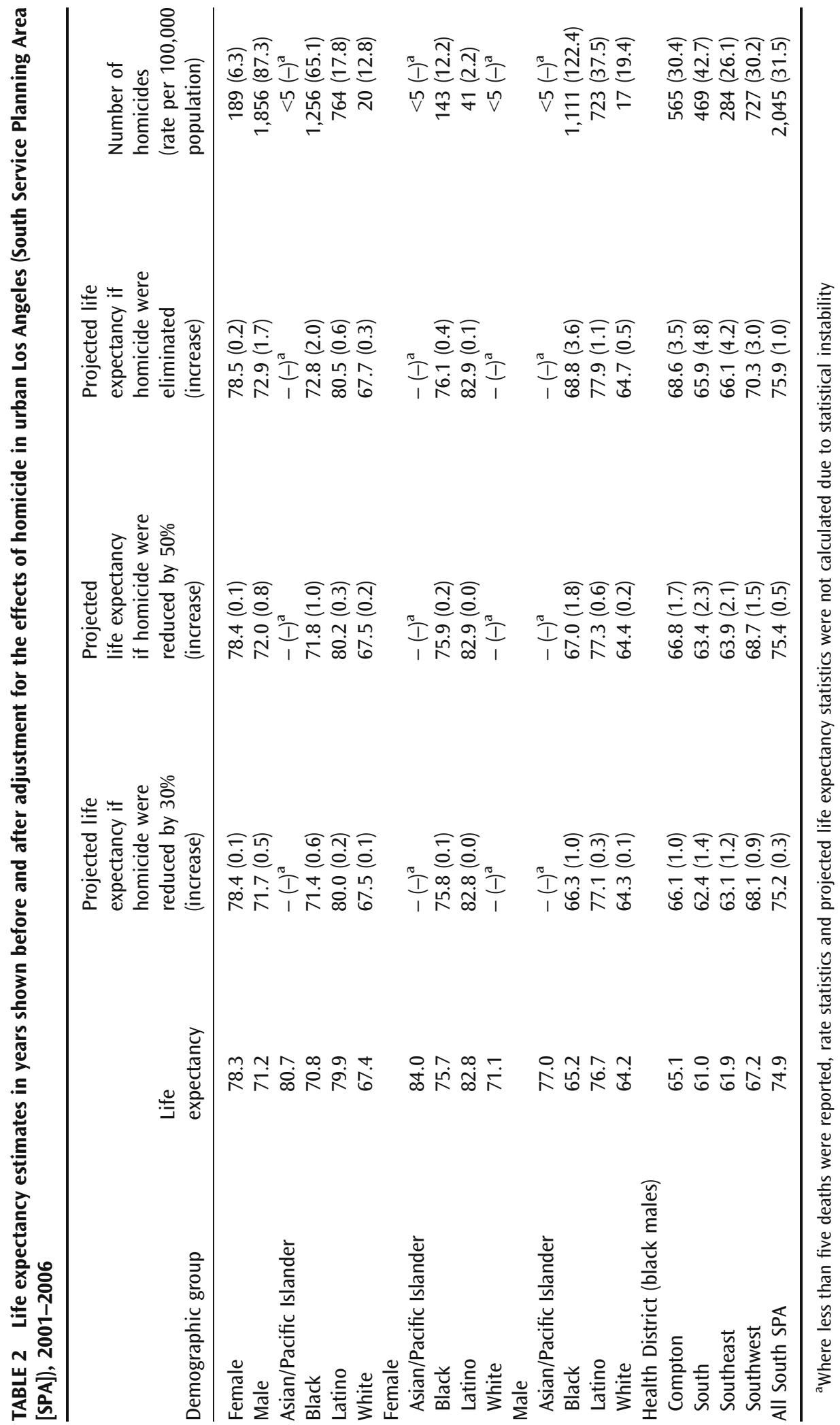


with strong social fabric can decrease levels of violent crime by providing a sense of social organization and belonging. ${ }^{10,13,19-22}$ A key factor in the success of Operation Ceasefire in Boston was the decision to address the culture of fear that led increasing numbers of youth to take dangerous measures to protect themselves. ${ }^{11}$ Homicide reduction efforts should include community-oriented policing and must involve community groups and leaders working together to build more cohesive social bonds within the community.

Cause elimination models contain the assumption that persons who died from homicide would have lived as long as other demographically similar LAC residents if homicide had been prevented. This may be incorrect. Some risk factors for homicide are tied to behaviors that negatively impact life expectancy in other ways, and if homicide were eliminated, would-be homicide victims might still die prematurely from other causes. Nevertheless, this assumption may hold more validity for homicide than it would for long-term chronic conditions such as heart disease or diabetes because homicide is a discrete event and is not correlated with a multitude of chronic illnesses which may soon cause death even if the potential death by homicide is averted. Additionally, it is unlikely that homicide rates could be reduced without addressing underlying behavioral and social risk factors. Thus, a reduction in homicide risk might also reduce the risk of death from associated behaviors.

Cause elimination models are an underutilized tool for measuring health disparities and mortality burden from homicide and other causes of death. This analysis demonstrates their utility. Similar methods have been used to assess the mortality burden from HIV ${ }^{2}$ and could be used to assess the mortality burden from other causes of death, particularly from discrete events such as accidents or other conditions like HIV which often occur in otherwise healthy individuals. Few publications have used homicide-adjusted life expectancy statistics and most have used them somewhat incidentally (e.g., as explanatory variables for other phenomena $)^{23-25}$ so that we are only aware of one published brief in the past 20 years that actually reports the impact of homicide on life expectancy. ${ }^{26}$ No published literature has reported the impact of homicide on life expectancy in urban areas. Instead, homicide data are typically presented as mortality rates or raw numbers of deaths. Data presented in these formats can be difficult to interpret and may obscure the magnitude of the problem. We believe that the full impact of homicide can be more clearly communicated to policy makers and members of the public by expressing that homicide takes two full years off the expected life span of black males in Los Angeles County and nearly 5 years off the expected life span of black males in parts of urban Los Angeles. This approach may be invaluable in assessing the impact of other events and diseases of public health importance.

OPEN ACCESS This article is distributed under the terms of the Creative Commons Attribution Noncommercial License which permits any noncommercial use, distribution, and reproduction in any medium, provided the original author(s) and source are credited.

\section{REFERENCES}

1. Los Angeles County Department of Public Health. Mortality in Los Angeles County 2005: leading causes of death and premature death. Los Angeles, CA: Los Angeles County Department of Public Health, Office of Health Assessment and Epidemiology; 2008. 
2. Lai D, Hardy RJ. Potential gains in life expectancy or years of potential life lost: impact of competing risks of death. Int J Epidemiol. 1999;28:894-898.

3. Arias E. United States life tables, 2004. National vital statistics reports, vol. 56 no. 9. Hyattsville: National Center for Health Statistics; 2007.

4. Data source: July 1, 2001-2006 Population and Poverty Estimates. Prepared by Walter R. McDonald \& Associates, Inc. (WRMA) for Urban Research, released 11/13/2007 (20012005) and 05/18/2007 (2006).

5. Chiang CL. Introduction to stochastic processes in biostatistics. New York: Wiley; 1968.

6. Chiang CL. Competing risks in mortality analysis. Annu Rev Public Health. 1991;12:281-307.

7. Ingram DD, Parker JD, Schenker N, et al. United States Census 2000 population with bridged race categories. National Center for Health Statistics. Vital Health Stat. 2003; 2(135):1-55.

8. Cubbin C, Pickle LW, Fingerhut L. Social context and geographic patterns of homicide among US black and white males. Am J Public Health. 2000;90(4):579-587.

9. Youth Gang Programs and Strategies. Office of Juvenile Justice and Delinquency Prevention. Washington, DC; 2000.

10. World Report on Violence and Health. World Health Organization. Geneva, Switzerland; 2000.

11. Kennedy DM, Braga AA, Piehl AM, Waring EJ. Reducing gun violence: the Boston Gun Project's Operation Ceasefire. Washington, DC: U.S. Department of Justice, National Institute of Justice, September 2001, NCJ 188741.

12. Reducing gun violence: operation Ceasefire in Los Angeles. Washington, DC: U.S. Department of Justice, National Institute of Justice, February 2005, NCJ 192378.

13. MacDonald JM. The effectiveness of community policing on reducing urban violence. Crime Delinq. 2002;48(4):592-618.

14. Wilkinson RG, Kawachi I, Kennedy BP. Mortality, the social environment, crime and violence. Soc Health Illness. 2008;20(5):578-97.

15. Kawachi I, Kennedy BP, Lochner K, Prothrow-Stith D. Social capital, income inequality, and mortality. Am J Public Heath. 1997;87(9):1491-8.

16. Robinson PL, Boscardin WJ, George SM, Teklehaimanot S, Heslin KC, Bluthenthal RN. The effect of urban street gang densities on small area homicide incidence in a large metropolitan county, 1994-2002. J Urban Health. 2009;86(4):511-2.

17. Kposowa AJ, Tsunokai GT, McElvain JP. Race and homicide in the US national longitudinal mortality study. J Ethnicity Crim Justice. 2006;4(1):1-27.

18. Kennedy BP, Kawachi I, Prothrow-Stith D. Income distribution and mortality: cross sectional study of the Robin Hood index in the United States. BMJ. 1996;312(7037):1004-7.

19. Kim-Ju G, Mark GY, Cohen R, Garcia-Santiago O, Nguyen P. Community mobilization and its application to youth violence prevention. Am J Prev Med. 2008;34(suppl 3):S5-12.

20. Payne PR, Williams KR. Building social capital through neighborhood mobilization: challenges and lessons learned. Am J Prev Med. 2008;34(suppl 3):S42-7.

21. Braga AA, Hureau D, Winship C. Losing faith? Police, black churches, and the resurgence of youth violence in Boston. Cambridge: Harvard University; 2008:1-28.

22. Braga AA. Pulling levers focused deterrence strategies and the prevention of gun homicide. J Crim Justice. 2008;36(4):332-43.

23. Kochanek KD, Maurer JD, Rosenberg HM. Why did black life expectancy decline from 1984 through 1989 in the United States? Am J Public Health. 1994;84(6):938-44.

24. Harper S, Lynch J, Burris S, Smith GD. Trends in the black-white life expectancy gap in the United States, 1983-2003. JAMA. 2007;297(11):1224-32.

25. Wilson W, Daly M. Life expectancy, economic inequality, homicide, and reproductive timing in Chicago neighbourhoods. BMJ. 1997;314(7089):1271-4.

26. Centers for Disease Control and P. Influence of homicide on racial disparity in life expectancy-United States 1998. JAMA. 2001;286(22):2805-6. 